\title{
Size Effect Of Electronic States In Graphene Quantum Disk
}

\author{
Xin $\mathrm{Li}^{\mathrm{a}}$, Xiaobo Feng ${ }^{\mathrm{b}}$ \\ School of Yunnan, Normal University, Yunnan 650500, China; \\ a359818751@qq.com, bfengxiaobo1220@gmail.com
}

Keywords: Graphene, quantum disk, Landau energy level

\begin{abstract}
In this paper, we studied the disk radius $\mathrm{R}$ graphene quantum dots of electronic spectroscopy by solving the Dirac equation of electron. We obtained two shapes of armrest and saw boundary of graphene quantum disk electronic energy level in the situation of whether with applied magnetic field or not, along with the change of radius of the quantum disk, respectively. The result of the research showed that the energy level of electron is inversely proportional to the radius of the quantum disk with no additional magnetic field. However, with the condition of additional magnetic field, the armchair quantum disk shows the least Landau energy level in degeneracy and it is proportional to radius. The energy level with positive value of quantum number $m$ of the zigzag quantum disk is proportional to radius and with negative value is inverse proportional to radius, which means the energy spectrum has sloped to the left.
\end{abstract}

\section{Introduction}

With modern chemical processing technology and development of materials synthesis process, people can easily obtain a new type of graphene materials by various means on the basis of graphene materials. It called graphene quantum dots, which has different shapes ${ }^{[1,2]}$ (disc, square, triangle, hexagon, etc.) and different sizes. In addition to the excellent properties ${ }^{[3-5]}$ of graphene $^{[6]}$ (high carrier mobility, quantum hall effect, Klein then heat wear and good chemical stability, etc.), graphene quantum dots also has several other special properties, such as quantum size effect, because of the limited carrier. So it has more attractive application prospect in many areas, such as solar photo-voltaic devices, aerospace materials, biological medicine, sensors and etc.

In recent years, the electronic energy state characteristics of graphene quantum dots material has become the focus of theoretical research.In 2010, Pan Hongzhe et.al have conducted a relative study on single equilateral triangle zigzag graphene quantum dots of electronic structure and magnetic made, founding the whole system of golden attributes. What is more, they found that dots on the electronic structure of the system effect is more obvious in smaller size of quantum. In 2011, Yao Zhidong studied the electronic properties of point defect armchair quantum disk graphene quantum dots by solving the Dirac equation. Results show that the lowest Landau degeneracy of energy level has nothing to do with the magnetic field strength, and a linear relationship with the radius. In 2013, He has made related research on the properties and electronic structure of graphene quantum dots of the triangular zigzag magnetic by constraint path quantum Monte Carlo calculation method and opportunity of the density functional theory numerical calculation method, and given the conclusion that in all dimensions of $\mathrm{ZZ}$ under ground state in the ferromagnetic state. Akola et.al have studied the electronic structure of graphene in the shape of specific zigzag size and armchair regular triangle with Tight Binding Method and Density Functional Theory. Rozhkow et.al have constructed a wave function dominated around the triangle structure, which studied electronic properties of graphene quantum dots of type armrest is triangle ${ }^{[7-11]}$. From the study of graphene electronic energy state in recent years, people is more of a concern and boundary conditions on the electronic energy state, the influence of the size of its cold can affect the attitude of the related research is reduced.

In this article, with the method of numerical solution of Dirac equation, we calculate graphene quantum dots of energy structure in zigzag and handrail type two kinds of boundary conditions of different size disc, respectively, with whether the vertical magnetic field or not. The calculation 
results of disk type of graphene quantum dots in the practical application have certain guiding significance.

\section{Theoretical model}

We consider a single graphene quantum disk which radius is $\mathrm{R}$, the Hamiltonian in the vertical magnetic field can be expressed as ${ }^{[12]}$

$$
H=v_{F}(\mathbf{P}+e \mathbf{A}) \cdot \sigma+\kappa V(r) \sigma_{z}
$$

The vF Fermi velocity and its size is about $106 \mathrm{~m} / \mathrm{s}$. The vector-potential of the external vertical magnetic field is $\mathrm{A}=A \mathrm{e}$, it has the relation with magnetic field intensity with $A=r B / 2 . \sigma$ is the Pauli matrix, $\kappa= \pm 1$ represent the position at point $K$ and $K^{\prime}$, respectively. By the infinite quality boundary condition, which is $V(r)=0(r<R)$, with $V(r)=\infty(r>R)$ 。In polar coordinates, the electron is confined by the Dirac equation $H \Psi(r, \phi)=E \Psi(r, \phi)$ the wave function is binary spin-or, corresponding to two nonidentical carbon atom in graphene quantum dots material, respectively. Considering the relation of electronic total angular momentum operator $J z$ with Hamiltonian $H,[H, J z]=0$, the wave function of system can be assumed ${ }^{[13]}$.

$$
\psi(r, \phi)=\frac{1}{\sqrt{r}}\left(\begin{array}{l}
-i R^{A}(r) e^{i m \phi} \\
R^{B}(r) e^{i(m+1) \phi}
\end{array}\right)
$$

$\mathrm{m}$ is the angular momentum,we can get a first order partial differential equation about radial direction couple interaction.

$$
\begin{gathered}
\frac{\partial}{\partial r} R^{B}(r)+\left(\frac{m+1 / 2}{r}+\frac{e B r}{2 \hbar}\right) R^{B}(r)=E R^{A}(r) \\
-\frac{\partial}{\partial r} R^{A}(r)+\left(\frac{m+1 / 2}{r}+\frac{e B r}{2 \hbar}\right) R^{A}(r)=E R^{B}(r)
\end{gathered}
$$

\section{Calculation method}

Without additional magnetic field, which means $\mathrm{B}=0$, the two types can be decomposed into two independent second order partial differential equations to obtain the anarchically solution; in another case, we use the finite difference method, the numerical solution to solve.Respectively from the armrest and zigzag boundary conditions to calculate.

For the boundary for armrest type material, the boundary condition is $R^{A}(r=0)=R^{B}(r=0)=R^{A}(r=R)=R^{B}(r=0)=0$, so we adopt central difference. (3) can be modeled as

$$
\begin{gathered}
\frac{R^{B}\left(r_{i}+1\right)-R^{B}\left(r_{i}-1\right)}{2 d r}+\left(\frac{m+1 / 2}{r_{i}}+\frac{e B r_{i}}{2 \hbar}\right) R^{B}\left(r_{i}\right)=E R^{A}\left(r_{i}\right) \\
-\frac{R^{A}\left(r_{i}+1\right)-R^{A}\left(r_{i}-1\right)}{2 d r}+\left(\frac{m+1 / 2}{r_{i}}+\frac{e B r_{i}}{2 \hbar}\right) R^{B}\left(r_{i}\right)=E R^{A}\left(r_{i}\right)
\end{gathered}
$$

Furthermore, 


$$
\left(\begin{array}{ccccccccc}
0 & a_{1} & 0 & \frac{1}{2 q} & 0 & 0 & \ldots & 0 & 0 \\
a_{1} & 0 & -\frac{1}{2 q} & 0 & 0 & 0 & \ldots & 0 & 0 \\
0 & -\frac{1}{2 q} & 0 & a_{2} & 0 & \frac{1}{2 q} & \ldots & 0 & 0 \\
\frac{1}{2 q} & 0 & a_{2} & 0 & -\frac{1}{2 q} & 0 & \ldots & 0 & 0 \\
0 & 0 & 0 & -\frac{1}{2 q} & 0 & a_{3} & \ldots & 0 & 0 \\
0 & 0 & \frac{1}{2 q} & 0 & a_{3} & 0 & \ldots & 0 & \frac{1}{2 q} \\
\vdots & \vdots & \vdots & \vdots & \vdots & \vdots & \ddots & -\frac{1}{2 q} & 0 \\
0 & 0 & 0 & 0 & 0 & 0 & -\frac{1}{2 q} & 0 & a_{n} \\
0 & 0 & 0 & 0 & 0 & \frac{1}{2 q} & 0 & a_{n} & 0
\end{array}\right) \times\left(\begin{array}{c}
R_{1}^{A} \\
R_{1}^{B} \\
R_{2}^{A} \\
R_{2}^{B} \\
R_{3}^{A} \\
R_{3}^{B} \\
\vdots \\
R_{n}^{A} \\
R_{n}^{B}
\end{array}\right)=\varepsilon\left(\begin{array}{c}
R_{1}^{A} \\
R_{1}^{B} \\
R_{2}^{A} \\
R_{2}^{B} \\
R_{3}^{A} \\
R_{3}^{B} \\
\vdots \\
R_{n}^{A} \\
R_{n}^{B}
\end{array}\right)
$$

Where $\varepsilon=E / \hbar v_{F}, a_{i}=\frac{m+1 / 2}{r_{i}}+\frac{e B r_{i}}{2 \hbar}$, $\mathrm{q}$ is the step length, $r_{i+1}-r_{i}=r_{i}-r_{i-1}$

For the boundary for zigzag type material, the boundary condition is $R^{A}(r=R)=R^{B}(r=0)=0$, discretized with finite difference method, and some for A component, using backward difference, for the B component, using forward difference, (3) can be discreted as, get it

$$
\begin{gathered}
\frac{R^{B}\left(r_{i}\right)-R^{B}\left(r_{i-1}\right)}{d r}+\left(\frac{m+1 / 2}{r_{i}}+\frac{e B r_{i}}{2 \hbar}\right) R^{B}\left(r_{i}\right)=E R^{A}\left(r_{i}\right) \\
-\frac{R^{A}\left(r_{i+1}\right)-R^{A}\left(r_{i}\right)}{d r}+\left(\frac{m+1 / 2}{r_{i}}+\frac{e B r_{i}}{2 \hbar}\right) R^{B}\left(r_{i}\right)=E R^{A}\left(r_{i}\right)
\end{gathered}
$$

Relatively,

$$
\left(\begin{array}{ccccccccc}
0 & a_{1}+\frac{1}{q} & 0 & 0 & 0 & 0 & \cdots & 0 & 0 \\
a_{1}+\frac{1}{q} & 0 & -\frac{1}{q} & 0 & 0 & 0 & \cdots & 0 & 0 \\
0 & -\frac{1}{q} & 0 & a_{2}+\frac{1}{q} & 0 & 0 & \cdots & 0 & 0 \\
0 & 0 & a_{2}+\frac{1}{q} & 0 & -\frac{1}{q} & 0 & \cdots & 0 & 0 \\
0 & 0 & 0 & -\frac{1}{q} & 0 & a_{3}+\frac{1}{q} & \cdots & 0 & 0 \\
0 & 0 & 0 & 0 & a_{3}+\frac{1}{q} & 0 & \cdots & 0 & 0 \\
\vdots & \vdots & \vdots & \vdots & \vdots & \vdots & \ddots & -\frac{1}{q} & 0 \\
0 & 0 & 0 & 0 & \ldots & 0 & -\frac{1}{q} & 0 & a_{n}+\frac{1}{q} \\
0 & 0 & 0 & 0 & 0 & \cdots & 0 & a_{n}+\frac{1}{q} & 0
\end{array}\right) \times\left(\begin{array}{c}
R_{1}^{A} \\
R_{1}^{B} \\
R_{2}^{A} \\
R_{2}^{B} \\
R_{3}^{A} \\
R_{3}^{B} \\
\vdots \\
R_{n}^{A} \\
R_{n}^{B}
\end{array}\right)=\varepsilon\left(\begin{array}{c}
R_{1}^{A} \\
R_{1}^{B} \\
R_{2}^{A} \\
R_{2}^{B} \\
R_{3}^{A} \\
R_{3}^{B} \\
\vdots \\
R_{n}^{A} \\
R_{n}^{B}
\end{array}\right)
$$

Diagonalizing (5) and (7) of matrix, we get each angular quantum number $\mathrm{m}$ of eigenvalue and eigenvector, namely the electronic energy and wave function.

\section{Discussion}

According to the numerical calculation method, we first of all, calculated under a single dimension $(\mathrm{R}=50 \mathrm{a}$, a is the distance between the carbon atoms, is about $0.142 \mathrm{~nm}$ ) quantum disk of the electron energy level in the absence of additional magnetic field, as shown in figure 1 . The 
figure shows that disc graphene quantum dots of electron energy spectrum related to angular quantum number $m$ division level, puts up two parts of the positive and negative energy, respectively corresponding to the electrons and holes. This feature is the same with the traditional material of semiconductor quantum dots. The difference is that the variation of graphene quantum dots is small, namely the interval between the energy levels were similar,while traditional level spacing of semiconductor quantum dots with quantum number increases. Armchair edged and zigzag edged under two kinds of boundary, electronic energy spectrum also has a big difference. For rail type boundary, the energy spectrum of quantum disk graph showing a certain symmetry, symmetry is shown as: (1) the positive and negative energy, namely electrons and holes symmetric energy; (2) with $\mathrm{m}=0$ and $\mathrm{m}=1$ as the axis of symmetry, spectrum bilateral symmetry. And zigzag graphene quantum disk this symmetry is broken, and the energy boundary can be 0 .

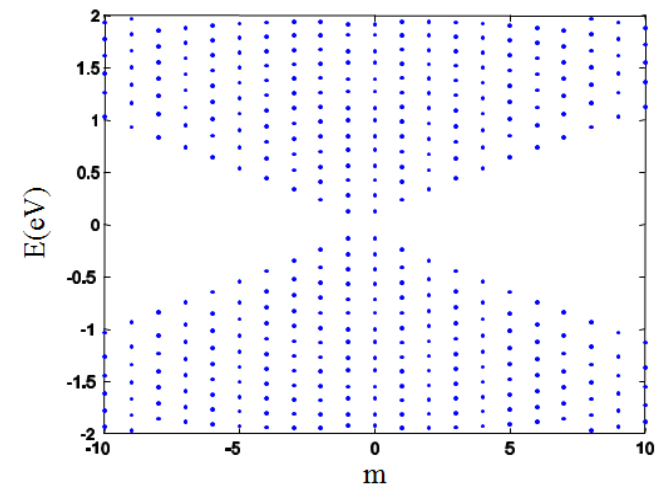

(a)

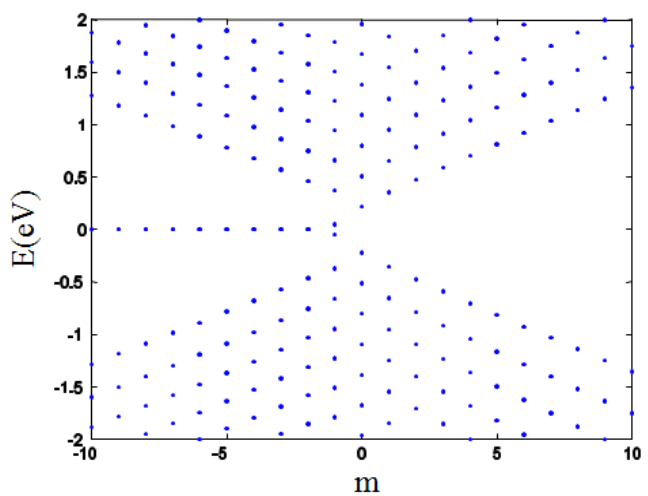

(b)

Fig. 1 Graphene quantum disk electron energy level(r=50a).

(a)armchair edged (b)zigzag edged

In order to study the effect of graphene quantum dot size on the level, we make no extra magnetic field angle of the quantum number $\mathrm{m}=0$ electronic level curve, along with the change of the quantum disk of radius $\mathrm{R}$, as shown in figure 2. Obviously, with the increase of the radius, the same quantum number of electron energy is reduced, thus narrow band gap. If the radius increases indefinitely, the highest level in the lowest energy of the electrons and holes will tend to overlap, the band gap disappeared, also is the condition of the single-layer graphene. By figure 3 can also see that the radius is small, the change of energy along with the change of the radius is obvious. With the traditional materials in the semiconductor quantum dots consistent quantum size effect.

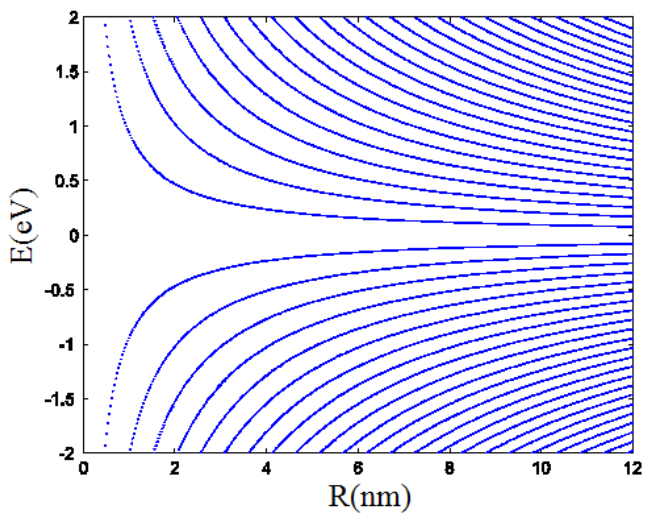

(a)

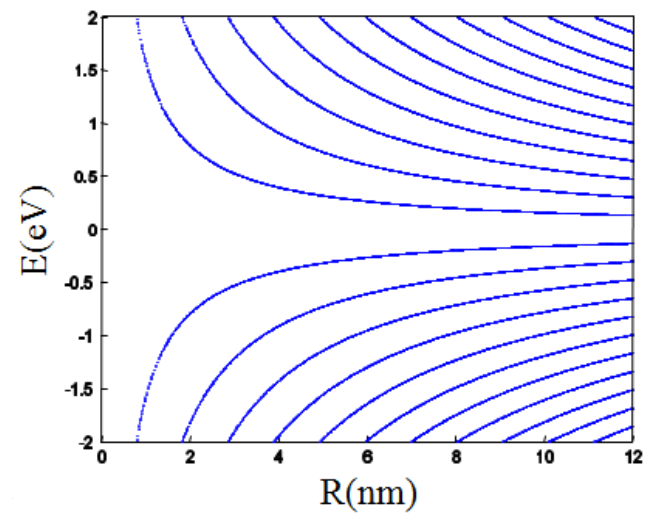

(b)

Fig. 2 The energy levels of graphene quantum disk radius.

(a)armchair edged (b)zigzag edged 


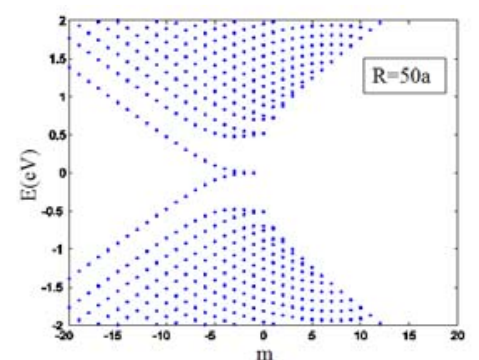

(a)

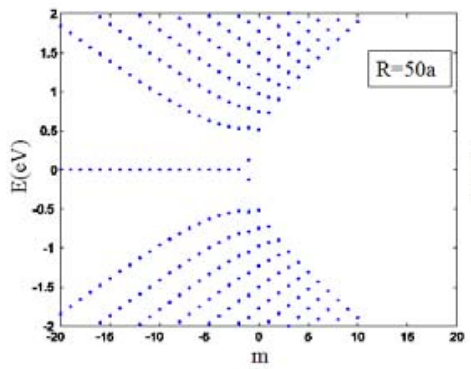

(d)

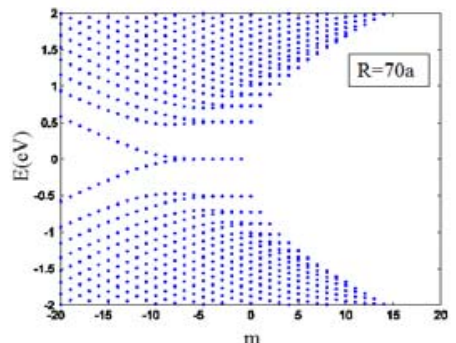

(b)

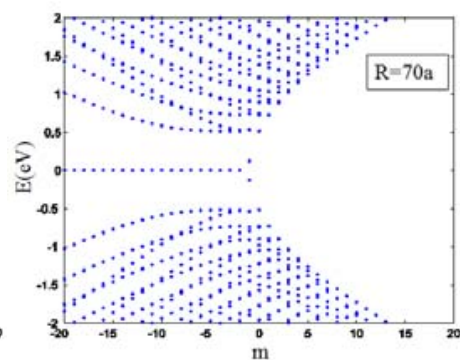

(e)

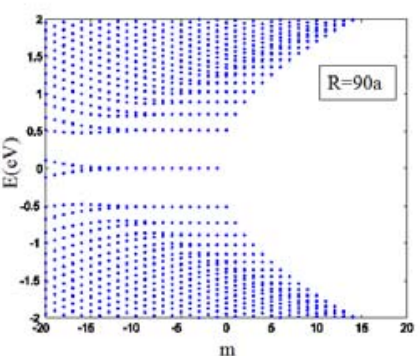

(c)

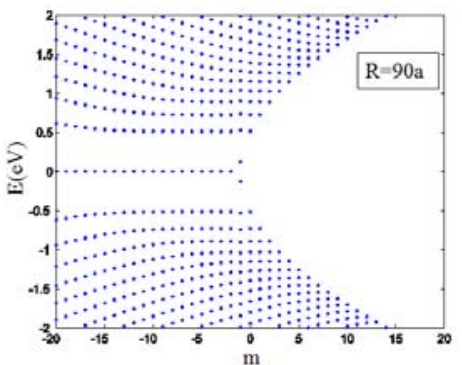

(f)

Fig. 3 Graphene quantum disk and magnetic field of the electronic energy spectrum diagram. (a)-(c))armchair edged(d)-(f))zigzag edged

Under the plus vertical magnetic field, the electronic energy spectrum of graphene quantum disk will change. With $\mathrm{B}=200 \mathrm{~T}$, figure 4 shows the energy spectrum when the radius is 50 a, 70 a, 90 a type of the armrest of and the zigzag quantum disk.Compared with the situation of figure 2 which without magnetic field, found that the armrest quantum disk type electronic energy spectrum of quantum number $\mathrm{m}$ about symmetry is broken, energy structure in the landau level of energy is 0 .And with the increase of size, with the number of zero energy state is increased also, the lowest landau degeneracy of energy level increased. And zigzag the change of the energy spectrum of quantum disk and disk is different. In the applied magnetic field, with the increase of radius of the quantum disk, the degeneracy of energy of 0 boundary states did not change, change other degeneracy of energy level.With the increase of the radius, non-zero degeneracy of energy level increased gradually. At the same time, the quantum number $\mathrm{m}$ positive energy state to reduce, $\mathrm{m}$ negative energy state, the energy spectrum appeared to the left of the tilt.

\section{Conclusion}

In this paper, through calculating numerical disk graphene quantum dot the Dirac equation for electronic movement, we obtained electron energy spectrum diagram under the armchair edged and zigzag edged two boundary of graphene quantum disk with whether external force field or not, and the electronic energy spectrum changing with the radius of the quantum disk. The study found that the disc graphene presents the discrete energy level; under the condition of no magnetic field, armrest the energy spectrum of quantum disk show the up-down symmetry and zygomorphy, whereas the zero energy in zigzag quantum disk boundary condition; With the increasing of radius of the quantum disk, two kinds of boundary of the quantum energy levels are lower, and the band gap become narrow; After Type in the vertical magnetic field, the armrest of quantum disk electron energy spectrum of quantum number $m$ about symmetry is broken, energy structure in the landau level of energy is 0 . With the increasing of the size, the lowest landau degeneracy of energy level increased. Under the condition of vertical magnetic field, the zigzag quantum disk with the increasing of the radius, the boundary of the energy of 0 mode degeneracy did not change, quantum number $\mathrm{m}$ positive energy state reduced, $\mathrm{m}$ negative energy state increased, the energy spectrum appeared to the left of the tilt. The study of graphene quantum dots in the design and production of photoelectric devices provides certain theoretical guidance. 


\section{Acknowledgement}

This research was supported by the National Natural Science Foundation of China (No. 1130427

5). The corresponding author is Xiaobo Feng.

\section{References}

[1]Ezawa M. Coulomb blockade in graphene nanodisks J. Phys. Rev. B 77(2008) 155411.

[2]Di C A, Wei D C, Yu G, Liu Y Q, Guo Y L, Zhu D B. Patterned Graphene as Source/Drain Electrodes for Bottom-Contact Organic Field-Effect Transistors J. Adv. Mater 20(2008) 3289-3293

[3]Novoselov K S , Geim A K, Morozov S V, Jiang D, Katsnelson M I, Grigorieva I V, Dubonos S V, Firsov A A. Two-Dimensional Gas of Massless Dirac Fermions in Graphene J. Nature 438(2005) 197-202.

[4]Zhang Y B, Tan Y W, Stormer H L, Kim P . Experimental Observation of Quantum Hall Effect and Berry's Phase in Graphene J. Nature 438(2005) 201-206.

[5]Nomura K, MacDonald A H. Quantum Hall Ferromagnetism in Graphene J. Phys. Rev. Lett. 96(2006) 256602-256605.

[6]Novoselov K S , Geim A K, Morozov S V, Jiang D, Zhang Y, Dubonos S V, Grigorieva I V, Firsov A A. Electric Field Effect in Atomically Thin Carbon Films J. Science 306(2004) 666-669.

[7]Pan H Z, Xu M, Chen L, Sun Y Y, Wang L. Electronic strcture and magnetism of single-layer trigonal graphene quantum dots with zigazag edges J. Chin.Phys.Soc.59(2010) 6443-6449.

[8]He B,Wang Z,Huang Z B.Effect of the size and edge reconstruction on magnetism in triangular graphene quantum dots with zigzag edges J. Mar.35(2010) 69-73..

[9]Yao Z D,Li W,Gao X L. Electeonic properties on the piont vacancy of armchair edged graphene quantum dots J. Acta phys.sin.61(2011) 117105.

[10]Akola J, Heiskanen H, Manninen M. Edge-dependent selection rules in magic triangular graphene flakes J. Phys. Rev. B 77(2008) 193410.

[11]Rozhkow A V, Nori F. Exact wave functions for an electron on a graphene triangular quantum dot J. Phys. Rev. B 81(2010) 155401.

[12]Schncz S, Ensslin K, Sigrisl M. Analytic model of the energy spectrum of a graphene quantum dot in a perpendicular magnetic field J. Phys. Rev. B 78(2008) 195427.

[13]Pereira V M, Nilsson J, Caslro Nelo A H. Coulomb Impurity Problem in Graphene J. Phys. Rev. Lell 99(2007) 166802. 